\title{
Die Frage der „Verträglichkeit“" innerstädtischer Einkaufszentren. Zum Mehrwert einer diskursanalytischen Perspektive auf raumbezogene Fragestellungen
}

\section{Towards a discourse and governmentality approach to spatial development - the case of inner city shopping centres}

https://doi.org/10.2478/rara-2020-0025

Eingegangen: 28. März 2020; Angenommen: 25. August 2020

Kurzfassung: Am Beispiel des politisch-planerischen Diskurses zu ehemaligen Planungen für ein großflächiges Einkaufszentrum in der Mainzer Innenstadt wird der potenzielle Mehrwert diskurs- und gouvernementalitätstheoretischer Zugänge zu raumbezogenen Fragestellungen erörtert. Insbesondere werden die diskursiven Mechanismen der Einschreibung einer unternehmerischen und depolitisierten Konzeption urbaner Räume in scheinbar neutrale und objektive Techniken raumbezogener Wirkungsanalysen herausgearbeitet. Die damit verbundene Sichtweise auf städtische Räume als Wettbewerbseinheiten, die durch raumwirtschaftliche Gesetzmäßigkeiten determiniert sind, wird jedoch gleichzeitig durch Gegendiskurse aufgebrochen, die das Bewahren ortsspezifisch gewachsener baulicher Strukturen zum Maßstab von Stadtentwicklungspolitik machen. Die Fallstudie verdeutlicht damit, wie mithilfe diskursanalytischer Zugänge die raumbezogenen Wissensordnungen freigelegt werden können, die die politischplanerische Steuerung gegenwärtiger Stadtentwicklungsprozesse anleiten.

Schlüsselwörter: Diskurs, Gouvernementalität, Kalkulation, Stadtentwicklung, Einkaufszentren, Innenstadtverträglichkeit, Mainz

Abstract: Using the example of the political-planning discourse on former plans to develop a large-scale shopping centre in the inner city of Mainz, the potential benefits of a discourse and governmentality approach to spatial research subjects is discussed. In particular, the discursive discursive mechanisms of inscribing an entrepreneurial and depoliticized conception of urban space into apparently neutral and objective techniques of spatial analysis are worked out. At the same time, this idea of urban space as a competitive entity determined by spatio-economic principles is challenged by competing conceptions of urban development focused on the conservation of place-specific

\footnotetext{
*Corresponding author: Dr. Denis Guth, Goethe-Universität Frankfurt am Main, Institut für Humangeographie, Theodor-W.-AdornoPlatz 6, 60323 Frankfurt am Main, Deutschland, E-Mail: guth@em.uni-frankfurt.de

Prof. Dr. Robert Pütz, Goethe-Universität Frankfurt am Main, Institut für Humangeographie, Theodor-W.-Adorno-Platz 6, 60323 Frankfurt am Main, Deutschland, E-Mail: puetz@em.uni-frankfurt.de
} 
structures of the built environment. Thus, the case study illustrates how discourse analysis can excavate the spatial knowledge structures guiding the management of contemporary urban development projects.

Keywords: Discourse, Governmentality, Calculation, Urban development, Shopping centres, Compatibility, Mainz

\section{Einleitung}

In der Zeitschrift „Raumforschung und Raumordnung | Spatial Research and Planning" hat Lintz (2017) eine wertvolle Debatte über die Bedeutung diskursanalytischer Zugänge zu raumbezogenen Fragestellungen angestoßen. Der vorliegende Beitrag möchte diese Diskussion weiter voranbringen und empirisch anreichern. Denn trotz einer gestiegenen Aufmerksamkeit für die Wechselwirkungen von Diskurs und Raum, so Lintz (2017: 319), seien diskursanalytische Ansätze in den Raumwissenschaften „bislang noch nicht (völlig) etabliert". ${ }^{1}$ Vielmehr gehe mit den poststrukturalistisch inspirierten, an Michel Foucault angelehnten Arbeiten ein gewisses Irritationspotenzial, wenn nicht gar ein „Unbehagen“ einher, das - so Lintz - zugunsten eines vertieften wechselseitigen Verständnisses überwunden werden sollte.

Wir greifen dies auf und plädieren dafür, diskursanalytische Zugänge weniger als konkurrierendes Paradigma zu raumwissenschaftlichen Ansätzen zu betrachten, sondern vielmehr als eine Bereicherung dahingehend, dass mit poststrukturalistisch inspirierten Theorien und Methoden klassische planungsrelevante Themen um neue Fragestellungen erweitert werden können. Hiervon können Impulse ausgehen, die zu einem tieferen Verständnis des Verhältnisses von Gesellschaft und Raum beitragen und dadurch auch praktisch relevant werden können.

1 Tatsächlich findet auch in der Humangeographie im Zuge des linguistic turn in den Sozialwissenschaften (vgl. Rorty 1967) bereits seit Längerem eine umfassende Rezeption diskurs- und gouvernementalitätstheoretischer Konzepte statt. Wichtige Bezugspunkte hierfür bilden unter anderen die Schriften Foucaults (vgl. einführend Dreyfus/Rabinow 1987), die diskurs- und hegemonietheoretische Arbeit von Laclau und Mouffe (2006) wie auch die Beiträge der governmentality studies (z. B. Miller/Rose 1990; Rose 1993; Bröckling/Krasmann/Lemke 2000). Auch wenn die Debatte im deutschsprachigen Raum angekommen ist (vgl. einführend Glasze/Mattissek 2009), spielt die Auseinandersetzung mit Diskursen in den traditionell stärker anwendungsorientierten Bereichen, die sich beispielsweise mit Planungsfragen oder auch der räumlichen Entwicklung im Einzelhandel befassen, allenfalls eine untergeordnete Rolle.
Unser Beitrag setzt einen Begriff ins Zentrum der Analyse, der in der politisch-planerischen Praxis räumlicher Entwicklung eine sehr wichtige Rolle einnimmt: „Verträglichkeit“. Ein städtebauliches Vorhaben ist in der gegenwärtigen Planungs- und Genehmigungspraxis nur dann genehmigungsfähig, wenn es seine „Raumverträglichkeit“ zu anderen Nutzungen nachweisen kann. Dies gilt in besonderem Maße für die Ansiedlung innerstädtischer Einkaufszentren, da sich hierbei die Frage nach der raumordnungs- und planungsrechtlichen „Verträglichkeit“" großflächiger Einzelhandelsvorhaben ${ }^{2}$ mit Fragen einer „baukulturellen und stadtstrukturellen Verträglichkeit“" (BMVBS 2011: 20) verschränkt. Eine Analyse konkreter Auseinandersetzungen um die Investitions„Verträglichkeit", die fast jedes Ansiedlungsvorhaben auf kommunaler Ebene begleiten, bietet damit das Potenzial, neben der (wissenschaftlich unterstützten) Suche nach möglichst geeigneten Verträglichkeits-Parametern die zusätzliche Fragestellung zu untersuchen, wie der Begriff der "Verträglichkeit" selber in lokal gebundenen Diskursen verhandelt wird: Wie wird "Verträglichkeit“ in konkreten Vorhaben von wem und von welchen machtvollen Positionen aus inhaltlich gefüllt, welche Bedeutung setzt sich im lokalen Diskurs durch und wie ist dies aus einer diskurstheoretischen Position heraus zu erklären? Dies sind Fragen, die für uns einen konkreten Mehrwert einer diskursanalytischen Herangehensweise an raumbezogene Fragestellungen greifbar machen können.

Hierfür skizzieren wir einführend zunächst die wesentlichen Konturen der überwiegend raumwissenschaftlich geprägten Debatte zur Frage der „Innenstadt-)Verträglichkeit“" innerstädtischer Einkaufszentren (Kapitel 2). Wie zu sehen sein wird, sind diese Arbeiten durch ihre Bemühungen um eine Versachlichung und Objektivierung der insbesondere ab Mitte der 1990erJahre vielfach kontrovers geführten Verträglichkeitsdebatte durch Ermittlung möglichst objektiver Verträglich-

2 In der gegenwärtigen Rechtsprechung und Praxis wird diese vorwiegend an die Frage schädlicher Auswirkungen auf zentrale Versorgungsbereiche gekoppelt, welche i.d.R. an Umsatzumverteilungsquoten auf der Grundlage gutachterlicher Kaufkraftstrommodellrechnungen festgemacht werden (Beckmann/ Lembke/von Schlippenbach et al. 2008: 73 f.). 
keitskriterien gekennzeichnet. Hieran anknüpfend gehen wir der Frage nach, inwieweit poststrukturalistische Konzepte von Diskurs und Gouvernementalität einen anderen Zugang zur Verträglichkeitsproblematik nahelegen können (Kapitel 3). Anhand der Ergebnisse eines von der Deutschen Forschungsgemeinschaft (DFG) geförderten Forschungsprojekts zu den Planungen eines ECE-Einkaufszentrums in der Mainzer Innenstadt möchten wir zeigen: Was als „verträglich“ gelten kann und was nicht, leitet sich weniger aus objektiv gegebenen ökonomischen und räumlichen Strukturen $a b$, sondern ist vielmehr davon abhängig, auf welche normativen Konzeptionen innerstädtischer Räume und auf welche Vorstellungen von einer "guten" Stadtentwicklungspolitik sich die politisch-planerische Praxis stützt. Hierfür untersuchen wir nach einer kurzen Vorstellung der Methodik der Fallstudie (Kapitel 4) zunächst die Problematisierungsmuster, die den Diskurs über die Verträglichkeit des ehemals geplanten Einkaufszentrums strukturieren (Kapitel 5.1). Anschließend betrachten wir die unterschiedlichen Steuerungslogiken zur politischplanerischen Bearbeitung der Verträglichkeitsproblematik, die hieraus hervorgehen (Kapitel 5.2). In Kapitel 6 diskutieren wir auf der Grundlage der Fallstudie mögliche Implikationen einer solchen diskursanalytischen Perspektive für die wissenschaftliche Auseinandersetzung mit der räumlichen Entwicklung von Städten.

Das Fallbeispiel zeigt, dass der Konflikt um die „Verträglichkeit“ innerstädtischer Einkaufszentren nicht nur als ein Konflikt zwischen unterschiedlichen Partikularinteressen beteiligter Akteure gelesen werden kann. Vielmehr wird ein wesentlich grundlegenderes Spannungsverhältnis sichtbar, das entlang der Frage verläuft, was den innerstädtischen Raum als solchen überhaupt ausmacht und welcher impliziten Logik die politisch-planerische Steuerung von Stadtentwicklungsprozessen folgt: Auf der einen Seite dieses Spannungsverhältnisses erscheint der innerstädtische Raum als Wettbewerbseinheit, die vermeintlichen Sachzwängen des Standortwettbewerbs ausgesetzt ist, abstrakten ökonomischen Gesetzmäßigkeiten unterliegt und deren Steuerung einer raumwirtschaftlich-funktionalen Optimierungslogik folgt. Auf der anderen Seite des Spannungsfeldes erscheint er vielmehr als eine ortsspezifisch gewachsene Struktur, die in ihrer Identität bewahrt und behutsam weiterentwickelt werden muss. Welche Konzeption des innerstädtischen Raums den politisch-planerischen Diskurs dominiert, ist dabei von zentraler Bedeutung für die Frage, wie innerstädtische Einkaufszentren „(innenstadt)verträglich" geplant werden können.

\section{Zur Frage der (Innenstadt-) Verträglichkeit innerstädtischer Einkaufszentren}

Insbesondere seit Mitte der 1990er-Jahre ist die Entwicklung innerstädtischer Räume in Deutschland durch eine erhebliche Zunahme einheitlich geplanter, entwickelter und betriebener Einkaufszentren geprägt. So hat sich die Zahl der Einkaufszentren von 93 im Jahr 1990 auf zuletzt $479 \mathrm{im}$ Jahr 2018 mehr als verfünffacht (EHI 2018: 189). ${ }^{3}$ Diese Zunahme ist zugleich mit einer deutlichen Verschiebung der vorrangigen Standortlagen von der "grünen Wiese" in die Innenstädte einhergegangen (EHI 2017: 190). Infolge dessen ist die Frage der „Innenstadt-)Verträglichkeit“" innerstädtischer Einkaufszentren zunehmend Gegenstand kontroverser raumwissenschaftlicher und stadtentwicklungspolitischer Debatten geworden. Die entsprechende Literatur umfasst neben Handreichungen für die kommunale Praxis (Beckmann/ Lembcke/von Schlippenbach et al. 2008; Junker/Kühn/ Pump-Uhlmann 2011; Fuhrich 2013) zahlreiche Beiträge der geographischen Handelsforschung, die die modelltheoretischen Ex-ante-Prognosen aus der gutachterlichen Begleitforschung um empirische Ex-post-Analysen zu den Auswirkungen innerstädtischer Einkaufszentren ergänzen (vgl. überblicksartig Weidner/Korzer 2014). Umfassende Synopsen der vorliegenden Studien, ihrer Forschungsdesigns und -ergebnisse wurden durch das Deutsche Seminar für Städtebau und Wirtschaft (DSSW) im Deutschen Verband für Wohnungswesen, Städtebau und Raumordnung e.V. erstellt (Beckmann/ Linnhoff 2012; Giesecke/Holl/Riedel et al. 2017), auf die weiterführend verwiesen werden kann. Charakteristisch für die darin aufbereiteten Studien ist, dass sie die Verträglichkeitsfrage in quantifizierbare, meist einzelhandelsgeographische oder immobilienwirtschaftliche Indikatoren übersetzen und dadurch operationalisierbar machen (vgl. hierzu die Übersicht in Tabelle 1). Hieraus sollen dann möglichst objektive Kriterien für eine „verträgliche" Shopping-Center-Entwicklung abgeleitet werden.

Durch diese Arbeiten konnte das Verständnis für die Wirkungszusammenhänge im Zuge der Ansiedlung innerstädtischer Einkaufszentren erheblich vertieft werden. Jedoch bleibt im Zuge der Objektivierungsbestrebungen zugleich ein wichtiger Aspekt unbeachtet:

3 Wobei die Dynamik ab den 2010er-Jahren bereits merklich nachgelassen hat und die Revitalisierung von Bestandsobjekten an Bedeutung gewinnt (EHI 2018: 189). 
Tabelle 1: In mindestens 15 Fallstudien herangezogene Indikatoren zur Bestimmung der Auswirkungen innerstädtischer Einkaufszentren

\begin{tabular}{|c|c|c|}
\hline Indikator & $\begin{array}{l}\text { Anzahl Fallanalysen I } \\
\text { Datensets }\end{array}$ & $\begin{array}{l}\text { Anzahl } \\
\text { Einkaufszentren }\end{array}$ \\
\hline Entwicklung der Einzelhandelsmietpreise (1A-Lage) & 96 & 66 \\
\hline Entwicklung der Zentralität/Zentralitätskennziffer der Standortgemeinde & 44 & 28 \\
\hline Entwicklung der Passantenfrequenzen (1A-Lage/Innenstadt) & 43 & 29 \\
\hline Umsatzentwicklung/Umsatzkennziffer der Standortgemeinde & 36 & 27 \\
\hline $\begin{array}{l}\text { Entwicklung des Anteils der Betriebe des modischen Bedarfs (Bekleidung/Schuhe) } \\
\text { in der } 1 \mathrm{~A} \text {-Lage/Innenstadt }\end{array}$ & 30 & 19 \\
\hline Kopplungsquoten Innenstadt und Einkaufszentrum & 27 & 22 \\
\hline $\begin{array}{l}\text { Entwicklung der Anzahl der Einzelhandelsbetriebe in der 1A-Lage/Innenstadt } \\
\text { (ohne Einkaufszentren) }\end{array}$ & 26 & 17 \\
\hline Entwicklung des Leerstandsanteils in der 1A-Lage/Innenstadt & 25 & 18 \\
\hline Lagestrukturveränderungen in der Innenstadt & 24 & 20 \\
\hline Entwicklung der innerstädtischen Einzelhandelsverkaufsfläche & 23 & 16 \\
\hline Entwicklung des Filialisierungsgrades in der 1A-Lage/Innenstadt & 21 & 18 \\
\hline Entwicklung des Einzelhandelsumsatzes in der Innenstadt (ohne Einkaufszentren) & 21 & 10 \\
\hline Entwicklung der wahrgenommenen Attraktivität der Innenstadt & 16 & 14 \\
\hline Veränderungen $1 \mathrm{~A}$-Lage & 15 & 13 \\
\hline Entwicklung der Besuchshäufigkeit der Kundinnen/Kunden in der Innenstadt & 15 & 14 \\
\hline
\end{tabular}

Quelle: modifiziert nach Beckmann/Linnhoff (2012: 34)

Jeder Versuch einer Antwort auf die Frage nach der "(Innenstadt-)Verträglichkeit" innerstädtischer Einkaufszentren setzt zunächst eine spezifische normative Konzeption von Innenstadt voraus. Das heißt, nur ausgehend von der Kontrastfolie einer ganz bestimmten Vorstellung davon, was innerstädtische Räume überhaupt ausmacht, was sie zukünftig sein sollen und welchen Zwecken sie dienen sollen, lässt sich beurteilen, ob ein Vorhaben mit dieser Vorstellung von Stadt "verträglich“ ist oder nicht. Es stellt sich somit die Frage, entlang welcher normativen Wissensordnung(en) über den innerstädtischen Raum die Auseinandersetzungen um die "Verträglichkeit“ innerstädtischer Einkaufszentren eigentlich ausgerichtet sind und welche Effekte für die politisch-planerische Steuerung von Stadtentwicklungsprozessen hieraus hervorgehen. Nachfolgend möchten wir darlegen, wie diese Frage mithilfe eines diskursanalytischen Zugangs näher beleuchtet werden kann.

\section{Die Frage der „Verträglichkeit“ aus diskurs- und gouvernemen- talitätstheoretischer Perspektive}

Die Verträglichkeitsfrage bildet den zentralen Kristallisationspunkt wissenschaftlicher und politisch-planerischer Auseinandersetzungen um geplante innerstädtische Einkaufszentren und deren Auswirkungen. Gleichzeitig erscheint der Verträglichkeitsbegriff trotz seiner engen planungsrechtlichen Rahmung facettenreich und entzieht sich einer endgültigen Fixierung. Die Deutungshoheit über die Frage, was „verträglich“ ist und was nicht, bleibt vielfach umkämpft. Umso wichtiger scheint es daher, näher zu untersuchen, wie der Verträglichkeitsbegriff im Zuge der konkreten Auseinandersetzungen um solche Ansiedlungsvorhaben tatsächlich inhaltlich gefüllt wird, welche raumbezogene Wissensordnung sich hierdurch um den Verträglichkeitsbegriff formiert und welche Machtwirkungen im Hinblick auf die politisch-planerische Steuerung von Stadtentwicklungsprozessen hieraus hervorgehen. Es geht also im Kern darum, zu rekonstru- 
ieren, wie die zentralen Begrifflichkeiten und Kategorien raumbezogener Wissensordnungen konstituiert werden, entlang derer konkrete stadtentwicklungspolitische Debatten und die politisch-planerische Steuerung von Stadtentwicklungsprozessen ausgerichtet werden.

Hierfür greifen wir auf das Konzept der „diskursiven Formationen“ zurück, das Michel Foucault in seiner „Archäologie des Wissens“ geprägt hat: „In dem Fall, wo man in einer bestimmten Zahl von Aussagen ein ähnliches System der Streuung beschreiben könnte, in dem Fall, in dem man bei den Objekten, den Typen der Äußerung, den Begriffen, den thematischen Entscheidungen eine Regelmäßigkeit (eine Ordnung, Korrelationen, Positionen und Abläufe, Transformationen) definieren könnte, wird man übereinstimmend sagen, daß man es mit einer diskursiven Formation zu tun hat" (Foucault 1981: 58).

Eine diskursive Formation ist durch spezifische Formationsregeln im Sinne empirisch beobachtbarer Regelmäßigkeiten innerhalb eines bestimmten Aussagefeldes (z. B. dem ökonomischen Diskurs, dem juristischen Diskurs oder dem Planungsdiskurs) gekennzeichnet. Ganz entscheidend ist hierbei, dass Foucault Diskurse nicht als sprachliche Abbilder oder Repräsentationen einer unabhängig davon gegebenen Wirklichkeit begreift, sondern als Praktiken, „die systematisch die Gegenstände bilden, von denen sie sprechen" (Foucault 1981: 74). Diskursanalysen im Anschluss an Foucault untersuchen entsprechend, wie eine solche Herstellung von Wirklichkeit erfolgt, und zwar durch die Regelhaftigkeit bestimmter sprachlicher Aussagefelder und „über Differenzbildungen und Bedeutungs- bzw. Sinnverkettungen“ (Keller 2011: 72). Dahinter steht ein poststrukturalistisches Verständnis von Sprache als differenzieller Verweisstruktur (vgl. Derrida 1976; Derrida 1990). Mit dieser Lesart erschließt sich beispielsweise der Bedeutungshorizont des Begriffs "Stadt" nicht aus einer vermeintlich gegebenen Essenz des Städtischen an sich, sondern erst aus den konkreten Verweisstrukturen auf andere Begriffe und aus den Differenzmarkierungen gegenüber dem „nicht Städtischen“, die erst im Diskurs hergestellt werden und historisch veränderlich sind.

Unsere zentrale Ausgangsthese lautet nun, dass die Auseinandersetzungen um die „(Innenstadt-)Verträglichkeit" innerstädtischer Einkaufszentren als Teil einer diskursiven Formation gelesen werden können - das heißt als eine Summe von auf den (innerstädtischen) Raum bezogenen Aussagen, die empirisch beobachtbaren Regelhaftigkeiten und Mechanismen folgen, die sie als "wahr" bzw. wahrheitsfähig produzieren. Was „Verträglichkeit“ für die politisch-planerische Praxis konkret bedeutet, ob ein geplantes Einkaufszentrum als „(innenstadt)verträglich“ gelten kann bzw. welche konkreten Interventionen dies erfordert, hängt aus einer solchen Perspektive nicht nur von ökonomischen, räumlichen oder städtebaulichen Gegebenheiten ab, sondern erscheint zentral auch als Effekt der dominierenden Verweisstrukturen zwischen innerstädtischem Raum und Center, die in stadtentwicklungspolitischen Diskursen etabliert werden.

Um dies zeigen zu können, legen wir zwei analytische Kategorien an den politisch-planerischen Diskurs zur „Verträglichkeit“ eines (ehemals) geplanten Einkaufszentrums in der Mainzer Innenstadt an, die Michel Foucaults (2004b, 2004a) Arbeiten zur Gouvernementalität entlehnt sind (Foucault 2004a; Foucault 2004b; vgl. auch Rose/Miller 1992: 178 ff. $)^{4}$. Dabei fragen wir erstens nach den spezifischen Problematisierungsweisen, die den Verträglichkeitsdiskurs strukturieren, und nach den Bezügen, die zwischen der Ansiedlung eines solchen Einkaufszentrums und der Entwicklung des innerstädtischen Raums dadurch hergestellt werden. Hierbei geht es um die grundlegende Frage, inwiefern die Ansiedlung eines innerstädtischen Einkaufszentrums überhaupt als etwas „Problembehaftetes“ verhandelt wird. Dies mag angesichts der zahlreichen potenziellen Konfliktlinien im Zuge solcher städtebaulicher Großvorhaben trivial erscheinen - ist es aber nicht. Entscheidend ist nämlich, wie und warum etwas als Problem verhandelt wird (Klöppel 2010: 255) und welche Zielvorstellungen und Risikoszenarien hierdurch mobilisiert werden (Mattissek 2008: 41). Problematisierung meint nicht einfach nur das Benennen gegebener Probleme, sondern die "kreative Arbeit“ (Lemke 1997: 343), die notwendig ist, um etwas auf ganz bestimmte Weise als Problem zu rahmen. Es besteht nämlich ein erheblicher Unterschied, ob die „verträgliche“ Entwicklung eines innerstädtischen Einkaufszentrums vorwiegend als ein ökonomisches, technisches, städtebauliches oder politisches Problem gerahmt wird - denn hierdurch können jeweils sehr unterschiedliche Bearbeitungsstrategien zur Bewältigung der Verträglichkeitsproblematik nahegelegt werden. Die Frage nach den dominierenden Problematisierungsmustern bildet somit einen „regulierende[n] Durchgangspunkt“" (Klöppel 2010: 260), denn sie stecken zugleich das diskursive Möglichkeitsfeld ab, innerhalb

4 Die governmentality studies untersuchen im Anschluss an Foucault, wie Wissens- und Diskursordnungen mit spezifischen Regierungsweisen und -techniken verschränkt sind. Gouvernementalitätsanalysen interessieren sich für das Verhältnis von Wissensproduktion und politischer Steuerung (Rose/Miller 1992). 
dessen eine politisch-planerische Bearbeitung der Verträglichkeitsproblematik stattfinden kann. ${ }^{5}$

Wir fragen daher zweitens, in Anlehnung an Foucaults Konzept der Rationalitäten des Regierens, nach den spezifischen Steuerungslogiken, welche die politisch-planerischen Interventionen zur Sicherstellung einer "verträglichen“ Shopping-Center-Ansiedlung anleiten. ${ }^{6}$ Dabei werden wir zeigen, dass Planungskonflikte im Zusammenhang mit der Verträglichkeitsfrage nicht nur akteurorientiert, nämlich als Konflikt gegenläufiger raumbezogener Partikularinteressen und Nutzungsansprüchen gelesen werden können. Vielmehr offenbart die diskursanalytische Betrachtung tiefergehende Bruchlinien und Widersprüche, die quer zu den beteiligten Akteuren und Institutionen verlaufen und auf unterschiedliche raumbezogene Wissensordnungen sowie auf grundlegend verschiedene Modi einer politisch-planerischen Steuerung der räumlichen Entwicklung von Innenstädten verweisen.

\section{Fallstudie und Methoden}

Als Fallstudie betrachten wir den Planungs- und Beteiligungsprozess für ein Einkaufszentrum an der Ludwigsstraße in Mainz. Nachdem bereits in den 1990er-Jahren Ideen für ein Einkaufszentrum in der Mainzer Innenstadt diskutiert worden waren, nahmen diese ab dem Jahr 2011 mit dem Erwerb des Karstadt-Komplexes aus Warenhaus, Nebengebäude und Parkhaus durch die ECE Projektmanagement konkretere Gestalt an. ECE plante die Errichtung eines innerstädtischen Einkaufszentrums mit rund $30.000 \mathrm{~m}^{2}$ Verkaufsfläche unter Einbezug des bestehenden Warenhausstandortes. Insgesamt sollten Investitionen von etwa 220 Mio. Euro rund 80 bis 90 Läden und 700 Arbeitsplätze schaffen (Landeshaupt-

5 Die Art der Problematisierung erschließt somit zugleich das Feld verfügbarer Bearbeitungsmodi mit ihren je spezifischen Wissensformen und Techniken.

6 Das Konzept der "Rationalitäten des Regierens“ ist in der Gouvernementalitätsliteratur mit derAnalyse der Transformation von Regierungsweisen auf der Makroebene verknüpft, insbesondere der Verschiebung von einer liberalen zu einer neoliberalen Gouvernementalität (Foucault 2004b). Für Analysen kleinteiliger Prozesse auf der Mikroebene, die nicht auf eine umfassende Gesamtanalyse gegenwärtiger Regierungsweisen abzielen, bevorzugen wir daher den Begriff der „Steuerungslogiken.“ Im Unterschied zu Strategien, die auf die Intentionalität von Akteuren abstellen, verweisen Steuerungslogiken auf das Wissen, das die politische Bearbeitung einzelner Problembereiche anleitet und mithilfe diskursanalytischer Zugänge untersucht werden kann. stadt Mainz 2012a: 46). Ausgelöst durch diese Planungen setzte eine Phase intensiver Wissensproduktion zu der Frage ein, wie das Einkaufszentrum „(innenstadt) verträglich" entwickelt werden kann. Diese wurde von teils kontroversen Auseinandersetzungen und der Entstehung einer Bürgerinitiative begleitet, die das Projekt in der geplanten Form verhindern wollte. Im Rahmen eines umfangreichen Partizipationsverfahrens veranstaltete die Stadt Mainz von Juni 2011 bis November 2013 insgesamt acht sogenannte „LudwigsstraßenForen“ („LuFos“). Hieraus sowie aus den Empfehlungen mehrerer Einzelhandels- und Verträglichkeitsgutachten sind konkrete Leitlinien für eine „verträgliche“ Shopping-Center-Entwicklung hervorgegangen, die durch Stadtratsbeschluss vom 24. Oktober 2012 zur Grundlage der Verhandlungen mit ECE gemacht und im Dezember 2013 an den Stand der Verhandlungen angepasst wurden (Landeshauptstadt Mainz 2013a). Letztere scheiterten jedoch in der Folge unter anderem an Grundstücksfragen, sodass die Planungen nicht umgesetzt werden konnten. Im Herbst 2016 zog sich ECE weitgehend aus dem Projekt zurück. Im Mittelpunkt des Prozesses steht seitdem die Erarbeitung eines neuen Nutzungskonzeptes jenseits klassischer Shopping-Center-Konzepte durch eine regionale Investorengruppe. Die vorliegende Analyse fokussiert insbesondere den Planungs- und Beteiligungsprozess der Jahre 2011 bis 2014, als die Frage der „(Innenstadt-) Verträglichkeit“" des damals geplanten Einkaufszentrums im Mittelpunkt stand.

Methodisch ist die Fallstudie als qualitative Diskursanalyse konzipiert, die sich kodierender Verfahren in Anlehnung an die qualitative Inhaltsanalyse bedient (Glasze/Husseini/Mose 2009) und softwaregestützt mithilfe des Programms MAXQDA durchgeführt wurde. ${ }^{7}$ Hierfür konnte ein umfangreicher Textkorpus aus den folgenden Dokumenten erstellt werden:

- Dokumentation des Beteiligungsprozesses im Rahmen von acht LudwigsstraßenForen (Protokolle, Präsentationen und Abschlussbericht inklusive 786

7 Kodierende Verfahren der qualitativen Inhaltsanalyse (vgl. Kuckartz 2014; Mayring 2015) haben sich insbesondere im Kontext des interpretativen Paradigmas der qualitativen Sozialforschung etabliert. Im Rahmen diskursanalytischer Untersuchungen sind daher Anpassungen an die theoretischen Vorannahmen erforderlich. So geht es weniger um die hermeneutische Interpretation der Sinnstrukturen einzelner Texte, die als in sich geschlossene Fälle untersucht werden. Im Mittelpunkt steht vielmehr die Beschreibung der Streuung von Aussagen innerhalb eines bestimmten diskursiven Feldes, also beispielsweise die Identifizierung wiederkehrender Problematisierungsmuster, die quer zu einzelnen Dokumenten und Akteuren verlaufen können. 
eingereichter Stellungnahmen und Anregungen beteiligter Akteure)

- 89 hieraus hervorgegangene Leitlinien und Empfehlungen in verschiedenen Fassungen (Entwurfsfassung, Beschlussfassung, Fortschreibungen im Laufe des Verhandlungsprozesses)

- Anträge und Anfragen, Beschlussvorlagen und Sitzungsprotokolle der städtischen Gremien (Stadtrat, Fachausschüsse, Ortsbeirat) einschließlich Anlagen (insgesamt 130 Dokumente)

- Drei Einzelhandels- bzw. Verträglichkeitsgutachten (bulwiengesa 2011; Junker und Kruse Stadtforschung Planung 2012; bulwiengesa 2014)

- Verhandlungsakten (1.215 Seiten) einschließlich Schriftverkehr und Protokollen zu den Verhandlungsrunden sowie unmittelbar hierauf bezogene Stellungnahmen diverser Akteure

- 31 Pressemeldungen der Stadt Mainz zur Erneuerung der Ludwigsstraße

- Informationsmaterialien der Bürgerinitiative Mainzer Ludwigsstraße e.V. mit Stellungnahmen zum Planungsstand

Zusätzlich wurden relevante Konzepte für die Steuerung der Einzelhandels- und Innenstadtentwicklung (z. B. Zentrenkonzept, Integriertes Entwicklungskonzept Innenstadt, Einzelhandelsmonitoring der Wirtschaftsförderung) sowie mehr als 350 relevante Presseartikel gesichtet. Der Korpus wurde durch ergänzende leitfadengestützte Interviews mit insgesamt zwölf Expertinnen und Experten angereichert, die das Projekt in unterschiedlichen Phasen und Rollen begleitet haben. Die Interviews bilden ein breites Spektrum beteiligter Akteure (unter anderem städtische Politik und Verwaltung, Gutachterinnen/Gutachter, Architektinnen/Architekten, Interessenvertretungen des Einzelhandels, Bürgerinitiative) mit inrem je spezifischen Zugang zu Stadtentwicklungsprozessen ab. Innerhalb der theoretisch hergeleiteten Analysedimensionen (Problematisierungsweisen, Steuerungslogiken) konnten durch induktives Kodieren die wesentlichen Diskursstränge im Sinne wiederkehrender Deutungs- und Argumentationsmuster herausgearbeitet werden, die den Verträglichkeitsdiskurs strukturieren.

\section{5 ,Verträglichkeit“" als Effekt raumbezogener Diskursordnung(en)}

Nachfolgend stellen wir die wichtigsten Ergebnisse der diskursanalytischen Fallstudie dar. ${ }^{8}$ Hierfür gehen wir zunächst auf die spezifischen Problematisierungsmuster ein, die den Verträglichkeitsdiskurs strukturieren. Im zweiten Schritt zeigen wir dann, welche politisch-planerischen Steuerungslogiken zur Sicherstellung von „Verträglichkeit" sich aus diesem diskursiven Möglichkeitsfeld heraus konstituieren. Abschließend möchten wir erörtern, welche möglichen Implikationen sich aus der Fallstudie ableiten lassen.

\subsection{Problematisierungsweisen der Ansiedlung innerstädtischer Einkaufszentren}

Unter dem Aspekt der Problematisierungsweisen haben wir danach gefragt, auf welche Weise die Realisierung eines Einkaufszentrums in der Mainzer Innenstadt in einen stadtentwicklungspolitischen Zusammenhang eingeordnet und dabei als etwas „Problembehaftetes" angesprochen wird. Es zeigt sich, dass das zentrale Motiv hierbei vermeintliche Handlungszwänge des Standortwettbewerbs waren. Die Verknüpfung erfolgte wie folgt: Der Verweis auf bestehende städtebauliche und funktionale Defizite des Vorhabenstandorts bildet den Ausgangspunkt dieses Problematisierungsmusters, das wir als "sachzwangorientiert" bezeichnen. Diese Defizite werden regelmäßig in Bezug gesetzt zum Argument eines sich zuspitzenden interkommunalen Standortwettbewerbs, dem sich die Stadt nicht entziehen könne. Deshalb sei „dafür Sorge zu tragen, dass die Stadt auch künftig im regionalen Wettbewerb insbesondere mit den rechtsrheinischen Städten ihre Attraktivität als Einkaufsstadt erhält, beziehungsweise ausbaut" (Landeshauptstadt Mainz 2011a: 2). In zahlreichen konkurrierenden Städten im Rhein-Main-Gebiet und in Rheinland-Pfalz seien zuvor bereits attraktive Einkaufszentren entstanden (Landeshauptstadt Mainz 2011a: 2). Mainz könne es sich daher, so der damalige Wirtschaftsdezernent, „nicht leisten, ein vergleichbares Produkt nicht zu haben“ (Renner 2011). Diese wenigen Diskursfragmente zeigen bereits sehr deutlich die zentrale Denkfigur des sachzwangorientierten Problematisierungsmusters: die Über-

8 Vgl. ausführlich Guth (2020). 
tragung einer unternehmerischen Handlungslogik auf die Steuerung der räumlichen Entwicklung von Städten. Diese müssen ihr „Angebotsprofil“ optimieren, um nicht ihre „Wettbewerbsfähigkeit" einzubüßen. Die Entwicklung von Innenstädten erscheint dabei als eine permanent notwendige Anpassungsleistung an äußere Sachzwänge des Standortwettbewerbs und geht mit einem stetig hohen Handlungsdruck einher: „Eines müssen sich aber alle Beteiligten nochmals bewusst machen: Keine Veränderungen an der Lu [Ludwigstraße] könnten in der Konsequenz Leerstand, Arbeitsplatzverlust und schließlich einen massiven Verlust an Attraktivität im Herzen der Stadt nach sich ziehen" (Landeshauptstadt Mainz 2011g: 4).

Vor diesem Hintergrund erscheint die Ansiedlung des geplanten innerstädtischen Einkaufszentrums als notwendiger und alternativloser Aufwertungsschub, um die identifizierten Defizite überwinden und die Wettbewerbsfähigkeit der Stadt sichern zu können: „Mainz braucht ein attraktives Einkaufszentrum in der Innenstadt zur Stärkung der Wettbewerbsfähigkeit des Einkaufsstandortes im regionalen Kontext, zur Verbesserung des Einzelhandelsangebotes für die Bevölkerung in Stadt und Region sowie zur städtebaulichen Aufwertung und Modernisierung des Ludwigsstraßen-Quartiers" (Landeshauptstadt Mainz 2012a: 82).

Das sachzwangorientierte Problematisierungsmuster ist eng verwoben mit einem zweiten Diskursstrang, der die Ansiedlung eines innerstädtischen Einkaufszentrums als Möglichkeit zur Aktivierung ungenutzter Potenziale anspricht. Dabei könne die Innenstadt als Ganzes von der Ansiedlung profitieren. Die Vielzahl der Interessen und Nutzungsansprüche an den innerstädtischen Raum lösen sich dabei scheinbar harmonisch in einem übergeordneten Gesamtinteresse an der Steigerung der Attraktivität und Wettbewerbsfähigkeit auf. Was dabei positiv als „Potenzial“ gerahmt wird, trägt jedoch zugleich das Notwendige in sich. Denn wenn die identifizierten Potenziale zur Attraktivierung der Innenstadt nicht ausgeschöpft werden, drohe ein weiterer Verlust von Wettbewerbsfähigkeit. Insofern sind potenzial- und sachzwangorientierte Problematisierungsmuster häufig direkt aufeinander bezogen.

Drittens ist der Verträglichkeitsdiskurs in erheblichem Maße durch den Verweis auf quantitative Indikatoren geprägt, die im Mittelpunkt eines ökonomischtechnokratischen Diskursstrangs über Zentralitäten, Bindungsquoten und Kaufkraftströme stehen. Eine wesentliche Rolle spielen hierbei Einzelhandels- und Verträglichkeitsgutachten, die in der Regel von spezialisierten Beratungsunternehmen und im Auftrag der betreffenden Ansiedlungskommune, häufig jedoch auf Kosten des Vorhabenträgers erstellt werden (Wolf 2012). Eine grundlegende Technik solcher Gutachten ist die Potenzialanalyse. Diese Technik wurde in Mainz bereits in einem frühen Planungsstadium im Rahmen zweier Gutachten (bulwiengesa 2011; Junker und Kruse Stadtforschung Planung 2012) aufgerufen, um zu beziffern, welches Potenzial für die Entwicklung zusätzlicher Verkaufsflächen in der Mainzer Innenstadt besteht. Die hieraus abgeleiteten Empfehlungen sollen einen ersten Orientierungsrahmen für die Verkaufsflächendimensionierung des geplanten Einkaufszentrums abstecken, die - neben städtebaulichen, verkehrsbezogenen und weiteren Fragestellungen - häufig ein zentrales Konfliktfeld bildet. Aus diskurstheoretischer Perspektive interessieren hierbei nicht nur die hieraus ermittelten Empfehlungswerte (die sich leicht unterhalb des von ECE geplanten Verkaufsflächenumfangs bewegen), sondern vor allem die Frage, wie diese hergeleitet werden. Hier zeigt sich, dass der entscheidende Schritt im Abgleich bestehender Zentralitätskennziffern mit Zielwerten liegt, die aus Vergleichen (benchmarkings) mit bundesweit ausgewählten Vergleichsstädten abgeleitet sind. Die Zentralitätsanstiege, die zur Erreichung der Zielwerte notwendig sind, werden dann über sortimentsspezifische Flächenproduktivitäten in Verkaufsflächenpotenziale umgerechnet. Unsere These lautet, dass durch die Anwendung solcher Quantifizierungstechniken letztlich mehr stattfindet als lediglich eine bloße Beschreibung bestehender Wettbewerbsverhältnisse: Durch die Verräumlichung betrieblicher Umsatzzahlen und das Benchmarking von Zentralitätswerten wird ein äußerer Bewertungshorizont aufgespannt, in dem Städte aufund absteigen können. Dabei werden Städte von ihrer spezifischen Sozialität, Historizität und Materialität entkoppelt, sodass sie als reine Wettbewerbseinheiten in einem abstrakten räumlichen Wirkungsfeld ökonomischer Variablen erscheinen können: „Modellierungen und Kalkulationen machen nicht einfach etwas sichtbar, das zuvor unsichtbar war - so als existierte eine direkte empirische Referenz der Repräsentation -, sondern sie verweisen auf die Arbeit an der Darstellung, die das, was sie darstellt, hervorbringt" (Kalthoff 2004: 171).

Wettbewerbsrelationen werden durch Benchmarking-Techniken somit nicht einfach nur abgebildet, sondern aktiv hergestellt (Larner/Le Heron 2005; Vormbusch 2007; Fougner 2008; Bruno 2009). Dabei ist die normative Vorannahme, dass Stadtentwicklung vorrangig an der Steigerung von Attraktivität und Wettbewerbsfähigkeit auszurichten sei, bereits in die institutionalisierten Kalkulationsweisen eingeschrieben, durch die 
(Innen-)Städte als Räume politisch-planerischer Interventionen lesbar gemacht werden. Die politische Frage, welche Art von Stadt eigentlich erstrebenswert ist, reduziert sich dann auf die technisch-ökonomische Frage, wie die Wettbewerbsfähigkeit des Standorts größtmöglich gesteigert werden kann. Technokratische Problematisierungen von "Verträglichkeit" schließen dabei zum einen semantisch an den potenzialorientierten Diskursstrang an, zum anderen konstituieren sie Städte diskursiv als Wettbewerbseinheiten und stabilisieren damit die dominierende Vorstellung von Stadtentwicklung als notwendiger Anpassungsleistung an äußere Sachzwänge des Standortwettbewerbs. Die Kalkulationstechniken, die im Rahmen des Verträglichkeitsdiskurses aufgerufen werden - so unsere These - können als Verdichtung einer raumbezogenen Wissensordnung gelesen werden, die Stadtentwicklung nicht als Ergebnis vielschichtiger, interner soziopolitischer Dynamiken begreift, sondern als technischen Optimierungsprozess, der an einem Referenzrahmen externer Wettbewerbsrelationen ausgerichtet ist und einer unternehmerischen Steuerungslogik folgt. In dieser Engführung von Stadtentwicklung auf einen scheinbar alternativlosen Wettbewerbs- und Optimierungszwang sehen wir eine der zentralen Machtwirkungen des Verträglichkeitsdiskurses.

Gleichzeitig verfestigen sich diese Problematisierungsmuster jedoch keineswegs zu einer vollständig in sich geschlossenen Diskursordnung. Vielmehr wird Letztere durch Gegendiskurse aufgebrochen, die aus einer solchen wettbewerbsorientierten Wissensordnung herausfallen. Diese stützen sich unserer Analyse nach insbesondere auf politisierende und/oder bedrohungsorientierte Problematisierungsmuster: Zum einen rahmen politisierende Problematisierungen den Planungsprozess als eine Arena gegenläufiger betriebswirtschaftlicher und „öffentlicher" bzw. „städtischer“ Interessen, die sich eben nicht konsensual auflösen ließen. Dieser Diskursstrang führt damit jedoch weg von der Verträglichkeitsfrage im engeren Sinne: Mainz benötige nichts, das "verträglich“ ist, sondern etwas, das "gut" für Mainz ist, so beispielsweise ein Mitglied des Ortbeirates MainzAltstadt im Rahmen der LudwigsstraßenForen (Landeshauptstadt Mainz 2012c: 39). Dies sei jedoch keine technische, sondern eine politische Frage. Im politisierenden Problematisierungsmuster geht es dabei nicht primär um die Steigerung der Wettbewerbsfähigkeit, sondern um etwas gänzlich anderes - nämlich um die Frage, wie in Anbetracht der Kapitalverwertungsinteressen durchsetzungsstarker institutioneller Investoren Einflussmöglichkeiten der planungsbetroffenen Bevölkerung auf die Stadtentwicklung gewahrt werden können. Somit ist dieser Diskursstrang nicht primär an ein technisch-ökonomisches Wissen, sondern an (Macht-)Fragen nach der Deutungs- und Gestaltungshoheit über den innerstädtischen Raum gebunden.

Zum anderen wird die oben beschriebene Diskursordnung durch bedrohungsorientierte Problematisierungen aufgebrochen. Dieser Diskursstrang stützt sich insbesondere auf das Motiv des Fremdkörpers, welches auf der Vorstellung einer grundlegenden Verschiedenheit und Unvereinbarkeit historisch gewachsener Städte auf der einen Seite und einheitlich geplanter Einkaufszentren auf der anderen Seite basiert. Exemplarisch wird das Fremdkörpermotiv anhand der nachfolgenden Sequenz aus einer schriftlichen Stellungnahme eines Mainzer Bürgers im Rahmen des Beteiligungsverfahrens greifbar: „Shoppingcenter haben nichts mit dem zu tun, was man das Wachsen einer Stadt nennt. Das Allmähliche, Prozeßhafte, Organische eines Wachstumsprozeßes wird durch den Überfall, das Statische, das Anorganische eliminiert: Die Notwendigkeit eines Shoppingcenters ist nie in dem Ort begründet, in dem es errichtet wird. Gelder des Finanzmarktes suchen egal wo eine Anlage und befallen im wahrsten Sinn des Wortes eine Stadt. Aus dieser Konstellation wird nie etwas anderes herauskommen als ein Fremdkörper" (Landeshauptstadt Mainz 2013b: 5).

Die Verortung innerstädtischer Einkaufszentren in einem semantischen Feld der Zerstörung („befallen“, „eliminieren“) stellt ein charakteristisches Merkmal des bedrohungsorientierten Diskursstranges dar und kann als eine Technik der Emotionalisierung und Mobilisierung von Widerstand gelesen werden, die in deutlichem Kontrast zu den bereits beschriebenen technokratischen Problematisierungsweisen und ihrem Anspruch auf „Sachlichkeit“ steht. Die hierbei evozierten Bedrohungsszenarien verdichten sich dabei zu Dystopien einer kommerzialisierten und verödeten Innenstadt, die nur noch den Konsum als urbane Lebenspraxis kennt und zulässt. Diesen Bedrohungsszenarien wird dann die Vorstellung einer besonderen Schutzbedürftigkeit gewachsener Strukturen und insbesondere der historischen Altstadt gegenübergestellt.

Diese Gegendiskurse werden insbesondere durch Projektkritikerinnen und -kritiker aus der Bürgerinitiative und dem Ortsbeirat getragen. Umgekehrt zeigen sich die oben beschriebenen vorherrschenden Problematisierungsmuster besonders deutlich in offiziellen Mitteilungen der Vorhabenträgerin und der Stadt Mainz zu dem geplanten Projekt. Eine Gleichsetzung der beschriebenen Diskursstränge mit einzelnen Akteuren und Institutionen greift unseres Erachtens aber zu kurz: Wenn 
etwa die Projektgegnerinnen und -gegner argumentieren, dass doch gerade der inhabergeführte Einzelhandel und die kleinteiligen, gewachsenen Strukturen der Altstadt die hohe Attraktivität der Mainzer Innenstadt im Vergleich zu anderen Städten ausmachten, so reproduzieren sie damit zugleich die Vorstellung von Innenstädten als Wettbewerbseinheiten, die ihr "Angebotsprofil“ optimieren müssen. Und umgekehrt stellt das Motiv des Fremdkörpers keineswegs nur eine rhetorische Figur zur Verhinderung des Projekts dar, sondern bildet - wie wir in Kapitel 5.2 zeigen werden - im planerischen Diskurs einen wichtigen Referenzpunkt für die Frage, wie eine Center-Entwicklung „(innenstadt)verträglich“ ermöglicht werden kann.

Aus einer diskursanalytischen Betrachtung geht es nun nicht darum, diese unterschiedlichen Problematisierungsweisen als mehr oder weniger zutreffende Wirklichkeitsbeschreibungen aufzufassen und sie nach inrem jeweiligen Wahrheitsgehalt zu befragen. Stattdessen geht es um die Wahrheitseffekte, die aus der spezifischen Regelhaftigkeit diskursiver Rahmungen hervorgehen. Erst durch diese diskursive Regelhaftigkeit wird eine bestimmte Problemstruktur aufgeworfen, wird die Verträglichkeitsfrage als Gegenstand politisch-planerischer Interventionen auf spezifische Weise denkbar. So legen die vorherrschenden Problematisierungsmuster, die sich auf die Vorstellung äußerer Sachzwänge des interkommunalen Standortwettbewerbs, die Aktivierung ungenutzter Potenziale zur Attraktivitätssteigerung und die Modellierung raumwirtschaftlicher Indikatoren stützen, ein ganz bestimmtes normatives Verständnis von Stadtentwicklungspolitik nahe. Demnach muss sich eine "erfolgreiche“ Stadtentwicklungspolitik in erster Linie daran messen, ob sie zu einer Steigerung der Attraktivität und Wettbewerbsfähigkeit im interkommunalen Standortwettbewerb beitragen kann. Hierbei geht es vor allem darum, private Investoren zu gewinnen und ihre Investitionsvorhaben unterstützend zu begleiten, um dadurch „Aufwertungsimpulse“ zu generieren. Wie wir nachfolgend noch ausführlicher zeigen werden, leitet sich aus dieser Wissensordnung eine Vorstellung von "Verträglichkeit" ab, die die räumliche Entwicklung von Innenstädten in ein betriebswirtschaftliches Optimierungsproblem übersetzt. Die Sicherstellung von "Verträglichkeit" zielt dann auf die Minimierung ökonomischer Umverteilungseffekte im inneren Bestand bei einer gleichzeitig größtmöglichen Attraktivitätssteigerung des Einzelhandelsstandortes nach außen. Es ist jedoch deutlich geworden, dass dieser wettbewerbsorientierte Diskurs mit verschiedenen Gegendiskursen konkurriert, die an gänzlich andere Vorstellungen von einer "guten“
Stadtentwicklungspolitik gebunden sind. Der politisierende Diskursstrang löst sich dabei von einer solchen Wettbewerbsorientierung und stellt stattdessen die Frage nach den Machtverhältnissen, innerhalb derer Stadtentwicklung stattfindet: Wer entwickelt den städtischen Raum und wessen Interessen werden dabei berücksichtigt? Damit einher geht die Forderung, die Stadtentwicklungspolitik dürfe sich nicht von den Kapitalverwertungsinteressen institutioneller Investoren abhängig machen. Stadtentwicklung sei nicht primär an Kriterien der Wettbewerbsfähigkeit auszurichten, sondern an Kriterien von Partizipation und demokratischer Legitimität. Vorrangige Maßgabe müsse daher die aktive Ausübung kommunaler Planungshoheit im Sinne des „Bürgerwillens" sein. Auch der bedrohungsorientierte Diskursstrang löst die Bindung von Stadtentwicklungspolitik an Kriterien der Wettbewerbsfähigkeit auf. An ihre Stelle lässt er eine Vorstellung von Stadtentwicklungspolitik als Bewahrung und behutsame Weiterentwicklung historisch gewachsener, identitätsstiftender Orte und Strukturen treten.

Die bisherige Analyse zeigt damit auch: Es greift erheblich zu kurz, die Auseinandersetzungen um die "Verträglichkeit" solcher Vorhaben lediglich als Konflikt zwischen unterschiedlichen Akteuren/Institutionen und ihren jeweiligen Partikularinteressen zu begreifen. Vielmehr lässt sich mithilfe eines diskursanalytischen Zugangs eine andere, tiefere Ebene solcher Planungskonflikte freilegen. So geht es bei der Verträglichkeitsfrage eben nicht nur darum, wie viel Verkaufsfläche ein Standort verträgt oder wie viele Ein- und Ausgänge ein Einkaufszentrum haben sollte - ,mitverhandelt' wird nämlich zugleich die wesentlich grundlegendere Frage, an welchen Kriterien die politisch-planerische Steuerung der Entwicklung innerstädtischer Räume überhaupt auszurichten ist.

\subsection{Steuerungslogiken zur Sicherstellung von „Verträglichkeit“}

Im zweiten Analyseschritt haben wir danach gefragt, auf welchen Rationalitätsstrukturen politisch-planerische Interventionen zur Sicherstellung von „Verträglichkeit" basieren. Die Fallstudie zeigt, dass die Steuerung der Verträglichkeitsproblematik keiner in sich geschlossenen Rationalität folgt, sondern vielmehr durch die Gleichzeitigkeit und das Zusammenwirken unterschiedlicher Steuerungslogiken gekennzeichnet ist, die sich aus dem beschriebenen diskursiven Möglichkeitsfeld heraus konstituieren: Optimieren, Vernetzen, Integrieren. Diese stützen sich auf je spezifische Techniken der Wissens- 
produktion und gehen mit unterschiedlichen normativen Vorstellungen von Stadtentwicklung einher.

Die Steuerungslogik des Optimierens schließt unmittelbar an das dominierende Problematisierungsmuster des Verträglichkeitsdiskurses an, welches zwischen vermeintlichen Handlungszwängen des Standortwettbewerbs und der Identifikation von Aufwertungspotenzialen aufgespannt und durch Techniken der Quantifizierung gestützt wird. Hierbei wird die Sicherstellung von „Verträglichkeit" als ein raumwirtschaftlich-funktionales Optimierungsproblem gerahmt, bei dem es darum geht, die Attraktivität des Einzelhandelsstandorts nach außen hin größtmöglich zu steigern, gleichzeitig aber die Umverteilungswirkung im Bestand zu minimieren. Die zentrale Stellschraube hierfür bildet die (sortimentsspezifische) Dimensionierung der Verkaufsflächen: „Zur Entfaltung einer positiven Magnetwirkung muss das Zentrum eine gewisse Mindestgröße und Attraktivität aufweisen, andererseits darf der Verkaufsflächenumfang nicht dazu führen, dass die Struktur der Innenstadt dauerhaft geschädigt wird“ (Landeshauptstadt Mainz 2012a: 82).

Hierbei wird wiederum eine betriebswirtschaftliche Optimierungslogik auf die Steuerung der räumlichen Entwicklung von Städten übertragen. Ziel ist die Bestimmung einer optimalen Verkauffflächengröße, ab deren Überschreitung der „Zusatznutzen“ einer weiteren Attraktivitätssteigerung nach außen durch interne Umverteilungseffekte überkompensiert würde. Es geht darum, „eine dauerhaft angemessene maximale Verkaufsfläche im EKZ verträglich zu entwickeln, um den Einzelhandel nicht weiter in seinem Bestand zu gefährden" (Landeshauptstadt Mainz 2011b: 2). Dafür komme es entscheidend darauf an, eine „Überdimensionierung“ zu vermeiden. Eine zentrale Funktion kommt daher den kalkulativen Techniken der Verträglichkeitsprüfung zu. Diese erstellen eine Prognose der erwarteten Umsatzumverteilung, die durch das geplante Einkaufszentrum ausgelöst wird, und rechnen diese mithilfe von Kaufkraftstrommodellrechnungen auf die einzelnen zentralen Versorgungsbereiche in einem zuvor definierten Einzugsgebiet um. ${ }^{9}$ Hierfür verwenden sie in der Regel probabilistische Gravitationsmodelle, die insbesondere auf Huff (1964) zurückgehen. Im Ergebnis machen diese Berechnungen dann beispielsweise sichtbar, in welchem Maß sich eine Erhöhung der Verkaufsfläche in einem bestimmten Sortimentsbereich durch die Ansiedlung eines innerstädtischen Einkaufszentrums in einer Erhö-

9 Zu methodischen Grundlagen von Verträglichkeitsgutachten vgl. ausführlich Beckmann/Lembcke/von Schlippenbach et al. (2008: $67 \mathrm{ff}$.$) .$ hung der insgesamt in der Innenstadt genierten Umsätze niederschlägt, andererseits aber mit Umverteilungen und Umsatzrückgängen im Bestand einhergeht. Auch hier lautet unsere These, dass solche Kalkulationsweisen mehr sind als eine reine Modellierung ökonomischer Realitäten. Vielmehr gehen sie mit einer grundlegenden Transformation der eigentlichen Problemstellung einher. Hierbei wird die Frage nach der angemessenen Dimensionierung eines Einkaufszentrums in der Mainzer Innenstadt - die ja konfliktbehaftet und politisch umkämpft ist - in eine wissenschaftlich-technische Fragestellung übersetzt, die durch raumwirtschaftliche Modellierungen zugunsten eines übergeordneten Gesamtinteresses der Wettbewerbsfähigkeit der Stadt scheinbar objektiv ,gelöst' werden kann. Damit wird eine Wissensordnung hervorgebracht, die innerstädtische Räume als unternehmerische Wettbewerbseinheiten innerhalb eines abstrakten Wirkungsfelds ökonomischer Variablen und statistischer Zusammenhänge in Erscheinung treten lässt.

Von dieser Optimierungslogik lässt sich eine zweite Steuerungslogik unterscheiden, die "Verträglichkeit“ durch eine intensive Vernetzung von Stadt und Einkaufszentrum sicherstellen will. Der zentrale Steuerungsansatz besteht dabei nicht in der Verkaufsflächendimensionierung, sondern in der gezielten Anregung und Steuerung von Zirkulationen. Demnach soll das geplante Einkaufszentrum vorhandene Wegebeziehungen so aufnehmen, dass die Passantenzirkulation innerhalb einer tripolaren Einzelhandelsstruktur der Mainzer Innenstadt weiter angeregt wird und auch die übrigen Bereiche der Innenstadt von der Ansiedlung profitieren können (Landeshauptstadt Mainz 2011f: 4). Ziel ist die „Entfaltung größtmöglicher positiver Wechselwirkungen" zwischen dem Einkaufszentrum, der City und den angrenzenden Einkaufslagen (Landeshauptstadt Mainz 2012a: 83), woraus im Unterschied zur Steuerungslogik des raumwirtschaftlich-funktionalen Optimierens sehr konkrete Anforderungen an die materielle Produktion von Stadt abgeleitet werden: „Es sollte im Hinblick auf die Durchlässigkeit für die Passantenfrequenz und damit die Anbindung an die Altstadt eine entsprechende Bauform realisiert werden. Dies könnte etwa durch Passagen oder etwa eine offene, sich auf mehrere Teilgebäude erstreckende Center-Architektur gewährleistet werden. Die Geschäfte im EG des Centers sollten nicht nur von einer inneren Mall erreichbar sein, sondern auch von außen. Dadurch wird die Durchlässigkeit zwischen Center und Stadtraum erhöht" (Landeshauptstadt Mainz 2011e: 9). 
Eine ähnliche Logik spiegelt sich auch in der Art und Weise wider, wie verkehrsbezogene Aspekte im Zuge des Planungs- und Beteiligungsprozesses verhandelt werden. Hierbei tritt - neben Machbarkeitsfragen der Bewältigung zusätzlicher Kunden- und Andienungsverkehre - eine strategische Komponente zutage, die auf die Steuerung von Kundenströmen abzielt. Deutlich wird dies unter anderem im Umgang mit dem ruhenden Verkehr: „Um eine unerwünschte Sogwirkung zu vermeiden, unterliegt das Center-Parkhaus einer Kapazitätsbeschränkung von max. 400 Stellplätzen. Diese Vorgabe ist eine Prämisse zur flächigen Verteilung von Kunden und Kundenverkehren im motorisierten Individualverkehr" (Landeshauptstadt Mainz 2012b: 3).

Die Steuerungslogik der Vernetzung stützt sich insbesondere auf Techniken des Messens, Zählens und Kartierens, die den städtischen Raum als ein sich selbst regulierendes, relationales Geflecht von Strömen und Frequenzen sichtbar machen. Dabei bleibt sie jedoch unmittelbar auf die oben beschriebene raumwirtschaftliche Optimierungslogik bezogen und leitet sich stringent aus dieser ab. Die Anregung der Zirkulation zwischen Stadt und Einkaufszentrum stellt zugleich das wesentliche Instrument zur Begrenzung der Umverteilungswirkung im Bestand dar und wird somit zur Möglichkeitsbedingung für die Steigerung der Gravitationswirkung durch Entwicklung zusätzlicher Verkaufsflächen. Während die Steuerungslogik des Optimierens jedoch auf das Risikoszenario des Zentralitätsverlusts bzw. des Bedeutungsverlusts im Standortwettbewerb bezogen ist, ist die Logik der Vernetzung primär am Risikoszenario des "Staubsaugereffekts“ ausgerichtet. Zudem unterscheiden sich beide Steuerungslogiken in den Subjektivierungsweisen, die sie hervorbringen: Während die raumwirtschaftlich-funktionale Optimierungslogik Subjekte als Kaufkraftträgerinnen und -träger konzeptualisiert, deren räumliches Konsumverhalten gravitationstheoretischen Gesetzmäßigkeiten unterliegt, operiert die Vernetzungslogik mit der Figur der Passantinnen/Passanten.

Hiervon lässt sich eine dritte Steuerungslogik abgrenzen, die auf eine behutsame und bewahrende Integration in bestehende Strukturen abzielt. Demnach soll sich das geplante Einkaufszentrum „[s]tädtebaulich und funktionsräumlich ... in den innerstädtischen Organismus einpassen; es muss sich als Bestandteil der gebauten Umgebung verstehen und darf sich nicht als introvertierte Insellage präsentieren" (Landeshauptstadt Mainz 2011d: 5). Erforderlich sei „eine besondere Qualität der Einfügung" (Junker und Kruse Stadtforschung Planung 2012: 13) in die „kleinteilige netzartige Struktur“ (Landeshauptstadt Mainz 2012a: 62) der Altstadt. Der
Verträglichkeitsbegriff, der hierin angelegt ist, unterscheidet sich grundlegend von dem oben beschriebenen Verständnis von „Verträglichkeit“ als Optimierungsproblem, das von universellen raumwirtschaftlichen Gesetzmäßigkeiten bestimmt wird. Vielmehr erscheint „Verträglichkeit" hier als etwas Ortspezifisches, das nur durch die sensible Bezugnahme auf lokale Gegebenheiten hergestellt werden kann. Paradigmatisch kommt dies in der Vorstellung zum Ausdruck, „[d]ie Projektentwicklung soll ,mainzverträglich' sein - es muss ein für unsere Stadt typisches Einkaufszentrum/Einkaufsquartier entstehen" (Landeshauptstadt Mainz 2011c: 1). Die Steuerungslogik des behutsamen und bewahrenden Integrierens nimmt einerseits das Risikoszenario der Entstehung eines Fremdkörpers auf. Andererseits löst sie die damit einhergehende Annahme der wechselseitigen Fremdheit und Unvereinbarkeit von gewachsener Stadt und geplantem Einkaufszentrum zugleich jedoch in quantitativen und qualitativen Strukturkategorien auf, sodass beide als Variationen des Gleichen erscheinen können - nämlich als spezifische Konfigurationen von Blockstrukturen, Gebäudehöhen, Fassadenlängen und -materialien, die einander angeglichen werden können. Im Mittelpunkt steht dabei die „Bewahrung und Stärkung der historischen und stadträumlichen Qualitäten und Strukturen wie Wegenetze, Blicke, Körnung, Proportion, Maßstab etc." (Landeshauptstadt Mainz 2011h: 57). So heißt es in einer Pressemitteilung der Stadt Mainz zu dem städtebaulichen Konzept, das aus den Verhandlungen mit ECE hervorgegangen ist: „Das Ensemble aus fünf Einzelbaukörpern ist keine zusammenhängende bauliche Anlage, sondern tatsächlich ein städtisches Viertel. Alle Gebäude haben auch in den glasüberdeckten Gassen Außenfassaden, und die Straßenbeläge werden ähnlich oder gleich sein wie in der Ludwigsstraße und auf dem Bischofsplatz. Die Eingänge und deren Glasfassaden treten hinter die Baukörper zurück, der Gutenbergplatz wurde architektonisch aufgewertet und die Durchlässigkeit durch die Mietflächen durch Anordnung weiterer Eingänge und Ausgänge für den Passanten verbessert. Das neue Karstadthaus ist an prominenter Stelle zum Gutenbergplatz angeordnet und hat als fünfter alleinstehender Baukörper eine eigene Adresse" (Landeshauptstadt Mainz 2013c: 2 f.).

Während die Steuerungslogiken des Optimierens und Vernetzens mit spezifischen Subjektivierungsformen - Kaufkraftträgerinnen/Kaufkraftträger und Passantinnen/Passanten - einhergehen, stützt sich die Logik des Bewahrens auf eine Vorstellung von Stadt als baulicher Struktur, die weitgehend ohne Subjekte auszukommen scheint. Gleichzeitig erweist sich diese Logik 
als besonders brüchig, wobei die zentrale Bruchlinie entlang der Frage verläuft, worin denn überhaupt die zu bewahrenden Strukturen bestehen (und worin nicht). So ist die städtebauliche Situation an der südlichen Mainzer Ludwigsstraße seit den 1960er-Jahren von einer kammartigen Struktur mit mehreren Pavillongebäuden und öffentlichen Plätzen dazwischen geprägt (Metzendorf 2011). Diese Struktur sollte zugunsten einer vorgezogenen, einheitlichen Baukante mit Schaufensterfront und mehreren Eingängen aufgegeben werden. Offensichtlich folgt die Logik des Bewahrens einer strategischen Selektivität. Sie stößt dort an ihre Grenzen, wo die Überprägung vorhandener Strukturen als funktionales Erfordernis erscheint, um eine hinreichende „kritische Masse“ an Verkaufsfläche entwickeln zu können und die Ludwigsstraße als „Flaniermeile“ zu stärken. Somit bleibt auch die Logik des Bewahrens der dominierenden Logik raumwirtschaftlich-funktionaler Optimierung nachgeordnet und dem übergeordneten Ziel der Attraktivitätssteigerung verpflichtet.

Zusammenfassend stellt sich der Verträglichkeitsdiskurs somit als ein Diskurs dar, der Innenstadtentwicklung vorwiegend als einen technisch-ökonomischen Optimierungsprozess rahmt, welcher durch äußere Sachzwänge des Standortwettbewerbs determiniert scheint. Die empirisch beobachteten Gegendiskurse werden dabei entweder in modifizierter Form aufgenommen (etwa das Fremdkörpermotiv, das auf potenziell anzugleichende städtebauliche Integrationskriterien wie Materialität, Maßstab und Körnung heruntergebrochen wird) oder in der Vorstellung eines übergeordneten Gesamtinteresses an der Stärkung der Wettbewerbsfähigkeit des Standorts aufgelöst.

\section{Implikationen}

Welche Implikationen lassen sich nun aus einer solchen diskursanalytischen Annäherung an den Verträglichkeitsbegriff, wie sie in der Mainzer Fallstudie vorgenommen wurde, ableiten? Hierzu möchten wir drei Anregungen formulieren:

Das Fallbeispiel hat erstens gezeigt, dass - ungeachtet der engen juristischen Rahmung des Verträglichkeitsbegriffs - in der Praxis der politisch-planerischen Steuerung sehr unterschiedliche Konzeptionen einer „verträglichen“ Einzelhandels- und Innenstadtentwicklung relevant werden können. Diese bewegen sich zwischen der Modellierung universeller raumwirtschaftlicher Gesetzmäßigkeiten einerseits und der Bezugnahme auf ortsspezifisch gewachsene Besonderheiten anderer- seits („Mainzverträglichkeit“). Sie sind an je spezifische normative Konzeptionen von Innenstadtentwicklung gebunden und leiten unterschiedliche politisch-planerische Steuerungslogiken an. Dabei geht es nicht einfach um das „Nacherzählen“ von Diskursen, sondern um die durchaus relevante Frage, wie und warum sich bestimmte Konzeptionen von "Verträglichkeit“ im stadtentwicklungspolitischen Diskurs durchsetzen können und welche Wirkungen hiermit einhergehen.

Die Fallstudie zeigt zweitens, wie diskursorientierte Ansätze den Horizont erweitern können und über die Suche nach ,objektiven' Verträglichkeitskriterien hinausgehen. Denn wie gesehen, müssen eine ganze Reihe von Techniken mobilisiert werden, damit die Verträglichkeitsfrage überhaupt als eine objektivierbare Frage gedacht werden kann. Die hierbei aufgerufenen Kalkulationstechniken sind mehr als eine reine Modellierung raumwirtschaftlicher Gegebenheiten: Sie bringen eine sehr spezifische Wissensordnung hervor, die innerstädtische Räume aus ihrer spezifischen Ortsgebundenheit, Historizität, Sozialität und Materialität herauslöst, sodass sie als abstrakte Raumeinheiten erscheinen können. Gleichzeitig werden diese in einen Referenzrahmen externer Wettbewerbsrelationen eingeordnet, sodass Innenstädte diskursiv als Wettbewerbseinheiten konstituiert werden, die einer unternehmerischen Optimierungslogik folgen und deren Entwicklung durch raumwirtschaftliche Gesetzmäßigkeiten determiniert ist. Somit entfalten diese scheinbar neutralen Techniken der Wissensproduktion eine erhebliche Machtwirkung, die als „Einschreibung ökonomischen Denkens in die Regierung des Städtischen" (Rosol 2013: 138) beschrieben werden kann. Sie übersetzen die politische Frage, wie sich ein bestimmter städtischer Raum entwickeln soll, in ein technisch-ökonomisches Optimierungsproblem, das auf die Verbesserung der Position im interkommunalen Standortwettbewerb gerichtet ist. Konfliktbehaftete Fragen wie die „verträgliche“ Dimensionierung innerstädtischer Einkaufszentren, die sich erheblich auf die Entwicklung und das Erscheinungsbild einer Innenstadt auswirken können, werden dabei in quasiwissenschaftliche Fragestellungen übersetzt, die durch technische Expertise im Sinne eines übergeordneten Gesamtinteresses an der Stärkung der Wettbewerbsfähigkeit der Innenstadt scheinbar objektiv gelöst werden können.

Diskursanalytischen Arbeiten wird drittens mitunter eine gewisse Praxisferne attestiert, wie auch Lintz (2017: 324) feststellt: „Angesichts der herausfordernden theoretischen Grundlagen, den häufig gewählten Forschungsfragen und der Zurückhaltung beim Geben von Politikempfehlungen dürfte besonders unter Prak- 
tikern die Befürchtung bestehen, dass sich die konstruktivistisch-diskursanalytische Wissenschaft zu weit von den gesellschaftlichen Problemen entfernt und für die alltägliche Planungspraxis nicht mehr relevant ist." Diese Einschätzung kann unseres Erachtens abgemildert werden. Diskursanalytische Ansätze können sehr wohl auch für die planerische Praxis relevant sein, allerdings - hier stimmen wir Lintz (2017: 324) zu - ,in einem völlig anderen Sinne": Es geht nicht vorrangig um die Bereitstellung instrumentellen Wissens für die bestehende Praxis, sondern um das Aufzeigen der Ambivalenzen und Widersprüche, die durch die bisherige Praxis unbearbeitet bleiben. Das Beispiel des Verträglichkeitsdiskurses zeigt, dass die beschriebenen Mechanismen der Naturalisierung einer zunehmend alternativlos scheinenden unternehmerischen, wettbewerbsorientierten Stadtentwicklungspolitik und das damit verbundene Narrativ der notwendigen Anpassung an äußere Handlungszwänge des Standortwettbewerbs in einem offenkundigen Spannungsverhältnis stehen zur gleichzeitigen Ausweitung partizipativer Verfahren und dem damit verbundenen Mitgestaltungsversprechen im Sinne einer Koproduktion von Stadt. Etwas zugespitzt ließe sich die Frage formulieren: Was bedeutet es für die Mitwirkungsmöglichkeiten an der Entwicklung urbaner Räume, wenn die städtische Bevölkerung in den Expertendiskursen, die Stadtentwicklungsprozesse anleiten sollen, lediglich als Kaufkraftträgerinnen/-träger, als Passantinnen/ Passanten oder gar nicht (wie in vielen städtebaulichen Betrachtungen) vorkommt? Und wie könnte eine Wissensproduktion zur Frage "(innenstadt)verträglicher" Stadtentwicklungsprojekte aussehen, die der Vielfalt urbanen Lebens in der europäischen Stadt eine stärkere Stimme gibt, ohne sie auf das Tätigen von Konsumausgaben und das Gehen von A nach B zu reduzieren?

Förderhinweis: Gefördert durch die Deutsche Forschungsgemeinschaft (DFG) - Projektnummer 374134160

\section{Literatur}

Beckmann, R. M.; Lembcke, J.; von Schlippenbach, U.; Wiemken, T. (2008): Integration innerstädtischer Einkaufszentren. Qualitative Integrationskriterien und Steuerung von Verträglichkeitsgutachten. Berlin. = DSSW-Leitfaden, 57.

Beckmann, R. M.; Linnhoff, C. (2012): Wirkung von Einkaufszentren in der Innenstadt. Synoptische Aufbereitung vorliegender Studien. DSSW-Studie. Bonn.

BMVBS - Bundesministerium für Verkehr, Bau und Stadtentwicklung (2011): Weißbuch Innenstadt. Starke Zentren für unsere Städte und Gemeinden. Berlin.
Bröckling, U.; Krasmann, S.; Lemke, T. (Hrsg.) (2000): Gouvernementalität der Gegenwart. Studien zur Ökonomisierung des Sozialen. Frankfurt am Main.

Bruno, I. (2009): The "Indefinite Discipline" of Competitiveness Benchmarking as a Neoliberal Technology of Government. In: Minerva 47, 3, 261-280. doi: 10.1007/s11024-009-9128-0

bulwiengesa AG (2011): Potenzialanalyse für ein innerstädtisches Einkaufszentrum an der Ludwigsstraße in Mainz. München.

bulwiengesa AG (2014): Auswirkungsanalyse Innerstädtisches Einkaufsquartier Ludwigsstraße. Frankfurt am Main.

Derrida, J. (1976): Die Schrift und die Differenz. Frankfurt am Main.

Derrida, J. (1990): Die différance. In: Engelmann, P. (Hrsg.): Postmoderne und Dekonstruktion. Texte französischer Philosophen der Gegenwart. Stuttgart, 76-113.

Dreyfus, H. L.; Rabinow, P. (1987): Michel Foucault. Jenseits von Strukturalismus und Hermeneutik. Frankfurt am Main.

EHI Retail Institute (2017): EHI Handelsdaten Aktuell 2017. Köln.

EHI Retail Institute (2018): Shopping-Center Report 2018. Köln.

Foucault, M. (1981): Archäologie des Wissens. Frankfurt am Main.

Foucault, M. (2004a): Sicherheit, Territorium, Bevölkerung. Geschichte der Gouvernementalität I. Vorlesung am Collège de France 1977-1978. Frankfurt am Main.

Foucault, M. (2004b): Die Geburt der Biopolitik. Geschichte der Gouvernementalität II. Vorlesung am Collège de France 19781979. Frankfurt am Main.

Fougner, T. (2008): Neoliberal Governance of States: The Role of Competitiveness Indexing and Country Benchmarking. In: Millennium - Journal of International Studies 37, 2, 303-326. doi: 10.1177/0305829808097642

Fuhrich, M. (2013): Innenstädtische Einkaufszentren - Positionen und Interpretationen. Bonn. = BBSR-Analysen Kompakt 05/2013.

Giesecke, C.; Holl, S.; Riedel, D.; Wagner, M. (2017): Langzeitwirkungen großflächiger Einzelhandelsbetriebe. DSSW-Studie. Berlin.

Glasze, G.; Husseini, S.; Mose, J. (2009): Kodierende Verfahren in der Diskursforschung. In: Glasze, G.; Mattissek, A. (Hrsg.): Handbuch Diskurs und Raum. Theorien und Methoden für die Humangeographie sowie die sozial- und kulturwissenschaftliche Raumforschung. Bielefeld, 293-314.

Glasze, G.; Mattissek, A. (2009) (Hrsg.): Handbuch Diskurs und Raum. Theorien und Methoden für die Humangeographie sowie die sozial- und kulturwissenschaftliche Raumforschung. Bielefeld.

Guth, D. (2020): Zur Sicherstellung der „Verträglichkeit“ innerstädtischer Einkaufszentren. Raumbezogene Diskurs- und Kalkulationsordnungen am Beispiel der Mainzer Innenstadt. Würzburg. = Geographische Handelsforschung 29.

Huff, D. L. (1964): Defining and Estimating a Trading Area. In: Journal of Marketing 28, 3, 34-38. doi: 10.2307/1249154

Junker, R.; Kühn, G.; Pump-Uhlmann, H. (2011): Zum Umgang mit großen innerstädtischen Einkaufscentern. Arbeitshilfe, herausgegeben vom Ministerium für Wirtschaft, Energie, Bauen, Wohnen und Verkehr des Landes Nordrhein-Westfalen. Düsseldorf.

Junker und Kruse Stadtforschung Planung (2012): Einkaufsstandort Ludwigsstraße. Einzelhandel und Städtebau. http://www. mainz.de/microsite/lufo/medien/bindata/LuFo_Forum-V_Gutachten-Langfassung.pdf (15.07.2020). 
Kalthoff, H. (2004): Finanzwirtschaftliche Praxis und Wirtschaftstheorie. Skizze einer Soziologie ökonomischen Wissens. In: Zeitschrift für Soziologie 33, 2, 154-175. doi: 10.1515/zfsoz2004-0204

Keller, R. (2011): Diskursforschung. Eine Einführung für SozialwissenschaftlerInnen. Wiesbaden. $=$ Qualitative Sozialforschung 14.

Klöppel, U. (2010): Foucaults Konzept der Problematisierungsweise und die Analyse diskursiver Transformationen. In: Landwehr, A. (Hrsg.): Diskursiver Wandel. Wiesbaden, 255-263.

Kuckartz, U. (2014): Qualitative Inhaltsanalyse. Methoden, Praxis, Computerunterstützung. Weinheim.

Laclau, E.; Mouffe, C. (2006): Hegemonie und radikale Demokratie. Zur Dekonstruktion des Marxismus. Wien.

Landeshauptstadt Mainz (2011a): Antrag 0247/2011/1 zur Sitzung Stadtrat am 16.02.2011. Stärkung der Mainzer Innenstadt durch die Errichtung eines Einkaufszentrums mit Passage in der Ludwigsstraße (gem. Antrag SPD, Bündnis 90/Die Grünen, FDP). Mainz. https://bi.mainz.de/getfile.php?id=53781\&type=do\& (16.07.2020)

Landeshauptstadt Mainz (2011b): Antrag 1086/2011/1 zur Sitzung Stadtrat am 15.06.2011. Neues Einkaufszentrum in der Ludwigsstraße - Karstadt-Komplex: Zukunft gestalten in Mainz (ÖDP/Freie Wähler). Mainz. https://bi.mainz.de/vo0050. php?_kvonr=7950\&search=1 (16.07.2020).

Landeshauptstadt Mainz (2011c): Antrag 1577/2011/2 zur Sitzung Stadtrat am 28.09.2011. Gemeinsamer Änderungsantrag zum Antrag der CDU 1577/2011 Einkaufszentrum in der Ludwigisstraße (SPD, Bündnis 90/Die Grünen, FDP). Mainz. https:// bi. mainz.de/getfile.php?id=64689\&type=do\& $(16.07 .2020)$.

Landeshauptstadt Mainz (2011d): Arbeitspapier. Rahmenbedingungen und Städtebauliche Vorgaben zur Entwicklung des Einzelhandelsstandortes "Ludwigsstraße" - sowie Vorschläge zum Projektablauf. Mainz. https://www.mainz.de/microsite/lufo/medien/bindata/LuFo_Arbeitspapier_Mai2011.pdf (16.07.2020)

Landeshauptstadt Mainz (2011e): LudwigsstraßenForum. Anregungen. Auftaktforum am 21. Juni 2011, Teil 2. Mainz. http://www. mainz.de/microsite/lufo/medien/bindata/LuFo_Forum-I_Anregungen-nach-21Jun2011.pdf (16.07.2020).

Landeshauptstadt Mainz (2011f): LudwigsstraßenForum. Protokoll. Auftaktforum am 21. Juni 2011. Mainz. http://www.mainz.de/ microsite/lufo/medien/bindata/LuFo_Forum-I_Protokoll.pdf (16.07.2020)

Landeshauptstadt Mainz (2011g): „Lufo“-Auftakt: Aus dem Experiment lernen. Mainz.

Landeshauptstadt Mainz (2011h): LudwigsstraßenForum IV: Stadtgestalt/öffentlicher Raum, 20. Dezember 2011. Präsentation. Mainz. https://www.mainz.de/microsite/lufo/forum4/index.php (16.07.2020)

Landeshauptstadt Mainz (2012a): Abschlussbericht LudwigsstraßenForum. Leitlinien - Empfehlungen. Mainz. https://www. mainz.de/microsite/lufo/index.php\#c10 (15.07.2020).

Landeshauptstadt Mainz (2012b): Anlage 3 zu Beschlussvorschlag 1540/2012. Synopse - Leitlinien und Empfehlungen. https://bi. mainz.de/getfile. .php?id=80849\&type=do\&\#sea rch $=\% 221540 / 2012 \% 22(24.07 .2020)$.

Landeshauptstadt Mainz (2012c): Anlage Abschlussbericht LudwigsstraßenForum. Anregungen - Fragen - Stellungnahmen. Mainz. https://www.mainz.de/microsite/lufo/index.php\#c10 (16.07.2020)
Landeshauptstadt Mainz (2013a): Beschlussvorlage 1722/2013 zur Sitzung Stadtrat am 04.12.2013. Entwicklung des Einkaufsquartiers Ludwigsstraße (Verhandlungsstand, weitere Vorgehensweise) / Bauleitplanverfahren „A 262“ / Ä 45 (Aufstellung). Mainz. https://bi.mainz.de/getfile.php?id=97896\&type=do\& (15.07.2020).

Landeshauptstadt Mainz (2013b): LudwigsstraßenForum VIII: Weitere Stellungnahmen. http://www.mainz.de/microsite/lufo/ medien/bindata/LuFo_Forum-VIII_weitere-Stellungnahmen. pdf (16.07.2020).

Landeshauptstadt Mainz (2013c): Einkaufsquartier Ludwigsstraße. Planungskonzept zum Eckpunktepapier vorgestellt. Pressemitteilung vom 16. Oktober 2013

Larner, W.; Le Heron, R. (2005): Neo-liberalizing Spaces and Subjectivities. Reinventing New Zealand Universities. In: Organization 12, 6, 843-862. doi: 10.1177/1350508405057473

Lemke, T. (1997): Eine Kritik der politischen Vernunft. Foucaults Analyse der modernen Gouvernementalität. Berlin.

Lintz, G. (2017): Foucault statt Fürst? Gedanken zu einem an Bedeutung gewinnenden Paradigma. In: Raumforschung und Raumordnung | Spatial Research and Planning 75, 4, 319-325. doi: 10.1007/s13147-017-0490-3

Mattissek, A. (2008): Die neoliberale Stadt. Diskursive Repräsentationen im Stadtmarketing deutscher Großstädte. Bielefeld.

Mayring, P. (2015): Qualitative Inhaltsanalyse. Grundlagen und Techniken. Weinheim.

Metzendorf, R. (2011): Die Ludwigsstraße im Wandel der Zeiten. Von der „Grande Rue Napoléon“ zur „Lu“. In: Stadt Mainz (Hrsg): Mainz. Vierteljahreshefte für Kultur, Politik, Wirtschaft, Geschichte 31, 3, 36-45.

Miller, P.; Rose, N. (1990): Governing economic life. In: Economy and Society 19, 1, 1-31. doi: 10.1080/03085149000000001

Renner, C. (2011): Lu: Stadt fragt die Mainzer. In: Rhein-Zeitung vom 10. Mai 2011, o. S.

Rorty, R. (1967) (Hrsg.): The linguistic turn. Recent essays in philosophical method. Chicago.

Rose, N. (1993): Government, authority and expertise in advanced liberalism. In: Economy and Society 22, 3, 283-299. doi: 10.1080/03085149300000019

Rose, N.; Miller, P. (1992): Political Power beyond the State: Problematics of Government. In: The British Journal of Sociology 43, 2, 173-205. doi: 10.2307/591464

Rosol, M. (2013): Regieren (in) der neoliberalen Stadt. Foucaults Analyse des Neoliberalismus als Beitrag zur Stadtforschung. In: Geographische Zeitschrift 101, 3+4, 132-147.

Vormbusch, U. (2007): Die Kalkulation der Gesellschaft. In: Mennicken, A.; Vollmer, H. (Hrsg.): Zahlenwerk. Kalkulation, Organisation und Gesellschaft. Wiesbaden, 43-63. doi: 10.1007/9783-531-90449-8

Weidner, S.; Korzer, T. (2014): Auswirkungen innerstädtischer Shopping Center auf tradierte innerstädtische Einzelhandelsstrukturen. Langzeitstudie „Höfe am Brühl“ in Leipzig. In: Kulke, E.; Rauh, J. (Hrsg.): Das Shopping Center Phänomen. Aktuelle Entwicklungen und Wirkungen. Mannheim, 73-108. = Geographische Handelsforschung 22.

Wolf, M. (2012): Anforderungen an Einzelhandelsgutachten. In: Konze, H.; Wolf, M. (Hrsg.): Einzelhandel in Nordrhein-Westfalen planvoll steuern! Hannover, 114-134. = Arbeitsberichte der ARL 2. 